\title{
Mutations in NR5A1 and PIN1 associated with idiopathic hypogonadotropic hypogonadism
}

\author{
S.C. Hu ${ }^{1,2}$, J. Ye ${ }^{1}$, A.K. Fathi ${ }^{1}$, X. Fu ${ }^{1}$, S. Huang ${ }^{1}$, Q. Ning ${ }^{1}$ and X.P. Luo ${ }^{1}$ \\ ${ }^{1}$ Department of Pediatrics, Tongji Hospital, Tongji Medical College, \\ Huazhong University of Science and Technology, Wuhan, Hubei Province, \\ China \\ ${ }^{2}$ Department of Nephrology and Hematology, \\ Qingdao Women and Children Hospital, Qingdao, Shandong Province, China \\ Corresponding author: X.P. Luo \\ E-mail:xpluo@tjh.tjmu.edu.cn \\ Genet. Mol. Res. 11 (4): 4575-4584 (2012) \\ Received February 27, 2012 \\ Accepted June 22, 2012 \\ Published October 9, 2012 \\ DOI http://dx.doi.org/10.4238/2012.October.9.6
}

\begin{abstract}
We tested the hypothesis that mutations in NR5A1 and PIN1 cause disorders in gonadotropin-gonadal system development and function, throught direct DNA sequencing of the coding sequence and splice-sites of NR5A1 and PIN1 in 50 subjects with sporadic idiopathic hypogonadotropic hypogonadism. These patients were recruited from the Pediatrics section of Tongji Hospital, Tongji Medical College, in Wuhan, China. None of the affected subjects had clinical signs of adrenal insufficiency. The NR5A1 and PIN1 mutations were found in 7 of the 50 cases. These 7 individuals presented severely low serum concentrations of testosterone or of estradiol and gonadotropin. Adrenal insufficiency was not diagnosed in any of these patients. Consequently, NR5A1 and PIN1 mutations should be considered in idiopathic hypogonadotropic hypogonadism patients with normal karyotypes and without adrenal insufficiency.
\end{abstract}

Key words: Hypogonadotropic hypogonadism; Gene mutations; NR5A1; PIN1 


\section{INTRODUCTION}

Idiopathic hypogonadotropic hypogonadism (IHH) can present anosmia, which is known as Kallmann syndrome (KS) and is characterized by total or partial loss of olfaction (Hardelin and Dode, 2008). Although autoimmune, environmental, and genetic factors contribute to IHH, there is no known cause in the majority of cases. In the past decades, a number of genes have been identified in the pathogenesis of IHH. Nevertheless, identification of genetic causes of IHH has been difficult elusive. For example, mutations in GPR54, FGF8, FGFR, NELF, KAL1, GnRH, GnRHR, GnRH1, PROKR2, CHD7, LHRH, PROK2, $T A C 3, T A C R 3, S F 1$, and $D A X-1$ are associated with IHH. Still, these genetic defects account for only about $30 \%$ of all cases of IHH (Crowley et al., 2008). This suggests that there are additional unknown factors that remain to be identified. The orphan nuclear receptor steroidogenic factor 1 ( $S F 1)$, also called Ad4BP, is encoded by the NR5A1 gene and is an essential regulator of endocrine function and development. It regulates the expression of both gonadotropin hormones in the pituitary and steroidogenic enzymes in the gonad (Schimmer and White, 2010). Newborn mice lacking NR5A1 exhibited a complex endocrine phenotype, including adrenal and gonadal agenesis, impaired expression of pituitary gonadotropins, and absence of the ventromedial hypothalamic nucleus (Ingraham et al., 1994; Shinoda et al., 1995; Achermann et al., 2001). PIN1 is a peptidyl-prolyl cis-trans isomerase that catalyzes the isomerization of phosphorylated Ser/Thr-Pro peptide bonds. PIN1 knock-out mice show marked abnormalities in their reproductive development and function (Atchison and Means, 2003). Recently, the important developments of genetics have occurred in the disorder of sex development field.

\section{MATERIAL AND METHODS}

\section{Laboratory assays}

Evaluation of basal hormones in the hypothalamus-pituitary-gonad axis consists of measuring serum testosterone, estradiol, luteinizing hormone (LH), and follicle-stimulating hormone (FSH) by enzyme-linked immunosorbent assay. When gonadal hormones or gonadotropins are low or inappropriately low in relation to the testosterone or estradiol levels, it indicates impairment of the hypothalamic-pituitary axis. In such case, magnetic resonance imaging of the pituitary should be considered.

\section{Patients}

Over a 2-year period (2008-2010), we studied 50 adolescent patients with sporadic primary amenorrhea and IHH from our pediatric endocrine clinic or genetics laboratory. Neither adrenal insufficiency nor syndromic features were reported in any of the patients. Patients with normal chromosomes, gonadal dysgenesis, and obvious defects in androgen and estrogen biosynthesis and action were included. The phenotypic spectrum of patients included: 1) prepubertal testicular volume $<4 \mathrm{~mL}$, absence of secondary sexual features (e.g., deepening of the voice, axillary and facial hair growth, and decreased muscle mass), 2) females with little or no breast development and primary amenorrhea, 3) absence of chronic disease in patients with 
certain conditions (e.g., obesity, hypothyroidism). The patients' clinical data were collected, and the correlation between genotype and phenotype was analyzed.

\section{DNA amplification and sequence analysis}

Informed consent was obtained from participants in the study and their parents. The Ethics Committee of Tongji Hospital, affiliated with Tongji Medical College, Huazhong University of Science and Technology approved this study. Genomic DNA was extracted from peripheral blood leukocytes using a Tiangen Biotech RelaxGene Blood DNA System. The entire exons and splice sites of NR5A1 and PIN1 were amplified by polymerase chain reaction (PCR). Oligonucleotide primers were designed with the Primer 5.0 software (Table 1). After amplification, the PCR products were purified using Shrimp and Alkaline Phosphatase and Exonuclease (Fermentas Company). Sequencing of these genes was performed on PCRamplified target regions using the ABI Prism Dye Terminator Cycle Sequencing Kit and an ABI 96-capillary 3730xl DNA analyzer.

\begin{tabular}{|c|c|c|c|c|}
\hline Exon & Forward primer $\left(5^{\prime}-3^{\prime}\right)$ & Reverse primer $\left(5^{\prime}-3^{\prime}\right)$ & Annealing temperature $\left({ }^{\circ} \mathrm{C}\right)$ & Length (bp) \\
\hline SF1-Exon2-3 & gggcacagagaggggatta & aagagtcatctccatagggttca & 60.7 & 626 \\
\hline SF1-Exon4 & tgaaagggtgttgagcagg & tgaagccagtgggaaggat & 60.8 & 849 \\
\hline SF1-Exon5 & gtagatgggcacagagaggtta & ggctgctcacagagggttt & 60.8 & 431 \\
\hline SF1-Exon6 & cctgcacctccaatccatg & gccetcagacctttgttcact & 62.9 & 420 \\
\hline SF1-Exon7-1 & tggtgacgatggtggtgtt & ccaagcagcaggcaagtg & 62.6 & 699 \\
\hline SF1-Exon7-2 & ctgcccetgagttctgacac & aatgacccagcaccaccc & 63.0 & 821 \\
\hline SF1-Exon7-3 & gcttggggattgccactaa & aaagagggtctgcgtgcc & 60.7 & 765 \\
\hline PIN1-Exon1 & ccagectttcttctacccaat & agcaggggtaggttgtggac & 61.5 & 657 \\
\hline PIN1-Exon2 & ggatttgttgaatgaaggcg & gctcaccctcaaatgccag & 60.8 & 533 \\
\hline PIN1-Exon3 & cttgggtgtgggtgaggac & gatgccaggaagaaagtgct & 64.0 & 532 \\
\hline PIN1-Exon4 & cgctcagcactggcaataa & ccetccceacgacatctt & 60.3 & 818 \\
\hline
\end{tabular}

\section{RESULTS}

\section{Mutational analysis of NR5A1 and PIN1}

Direct sequencing of PIN1 from DNA of patient 1 identified a heterozygous c.94A $>\mathrm{T}$ mutation in exon 2 (Figure 1), which resulted in a serine (Ser) to cysteine (Cys) substitution in codon 32 (p.Ser32Cys). Patient 2 showed c.734T $>$ G variant (Figure 2). This mutation led to a leucine (Leu) to Tryptophan (Trp) substitution at codon 245(p.leu245Trp). We identified 4 new heterozygous NR5A1 mutations in patients 3, 4, 5, 6, and 7. Among them, 2 were nucleotide substitutions, c.437G $>\mathrm{C}$ (p.Gly146Ala), in patients 4 and 5 (Figure 3); 1 was a synonymous mutation, c.351C $>\mathrm{G}$ (p.Gly117Gly), in patient 3 (Figure 4). The other 2 NR5A1 mutations were in non-coding regions: at position $1655 \mathrm{C}>\mathrm{T}$ of patient 6 (Figure 5) and 2973 $\mathrm{T}>\mathrm{C}$ of patient 7 (Figure 6) in exon 7. Although DNA was not obtained from these patients' siblings and parents, they had apparently normal fertility and reproductive function. Restriction analysis of DNA from 100 racially matched control subjects did not identify any of these variants. 


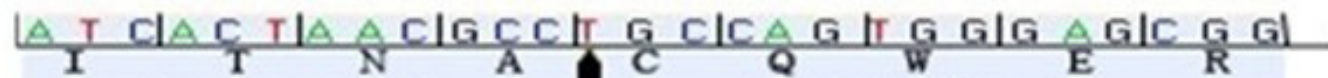
mutant exon2

C. A94T P. S32C

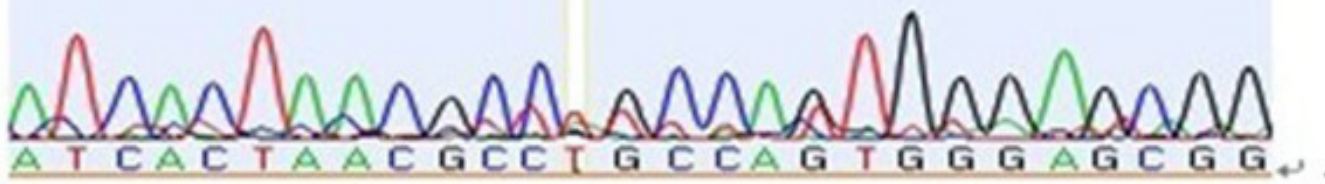

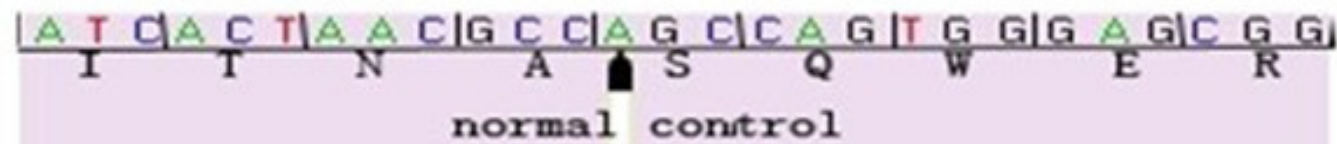

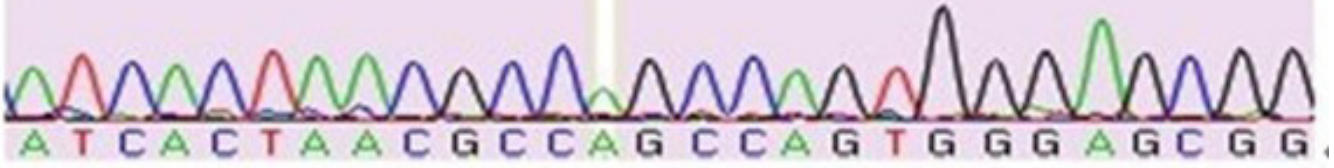

Figure 1. Patient 1 c.94AT(p.S32C).

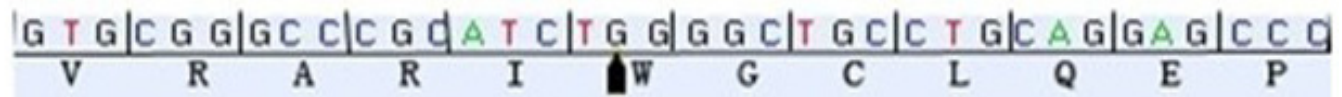
mutant C. T734G $\quad$ P. L245W

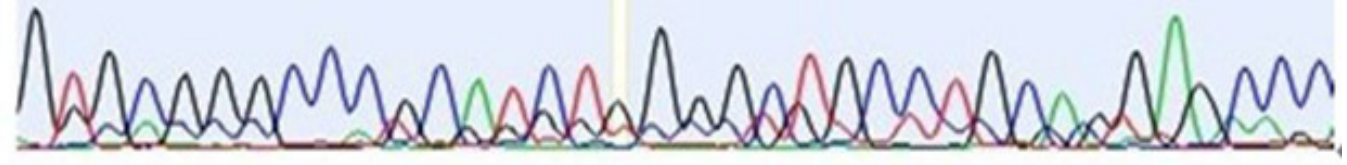

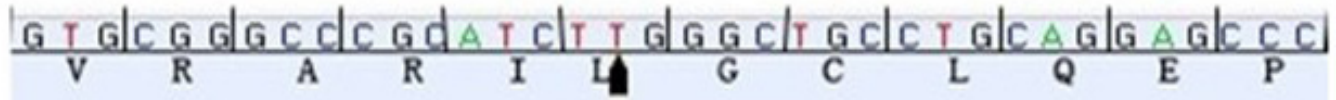
normal control

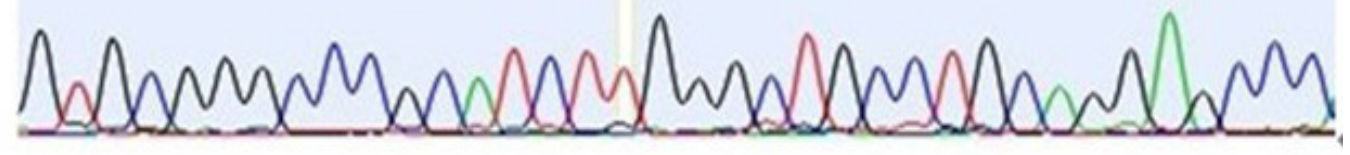

Figure 2. Patient 2 c.734TG(p.L245W). 


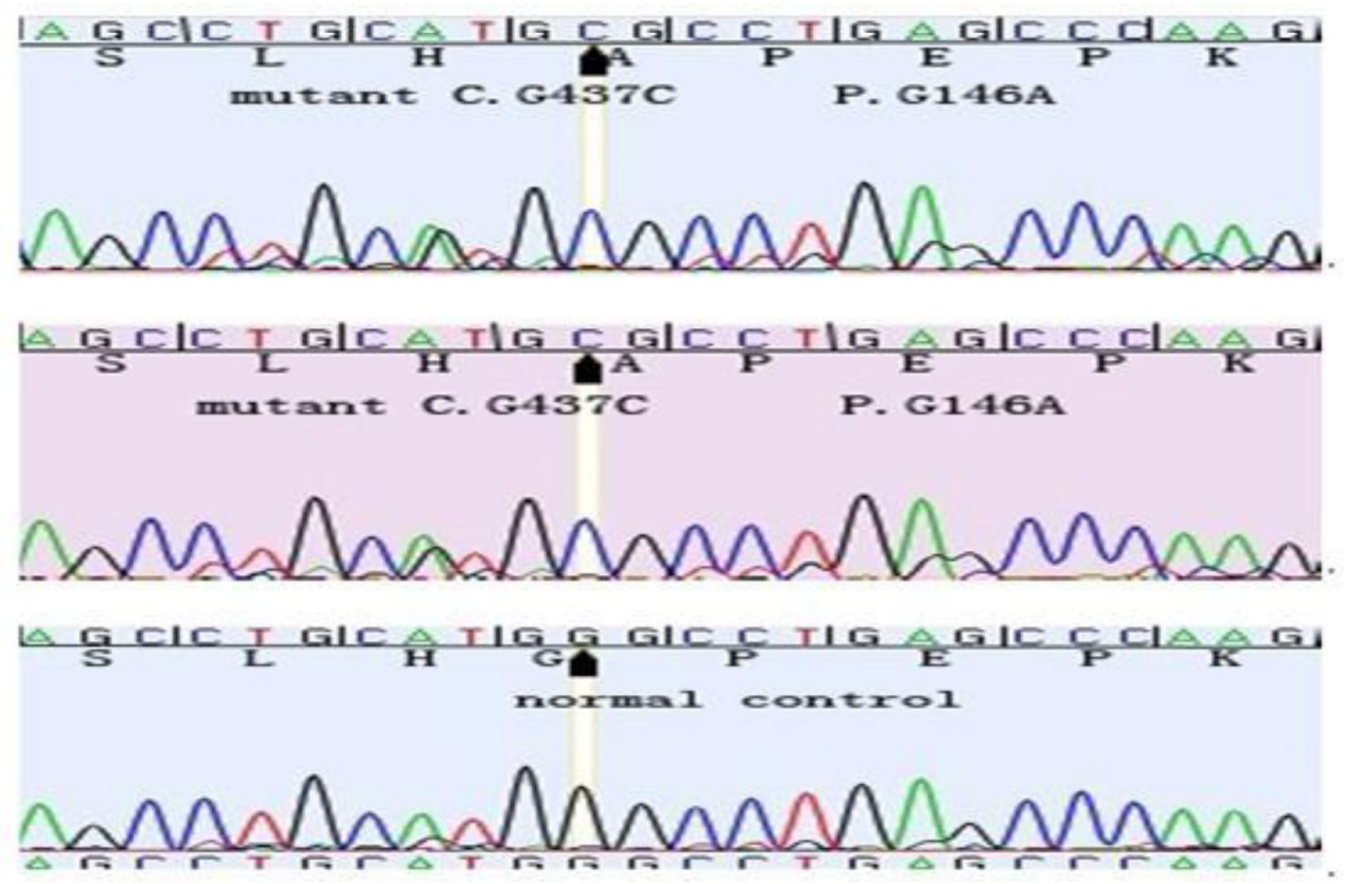

Figure 3. Patients 4 and 5 c.437GC(p.G146A).

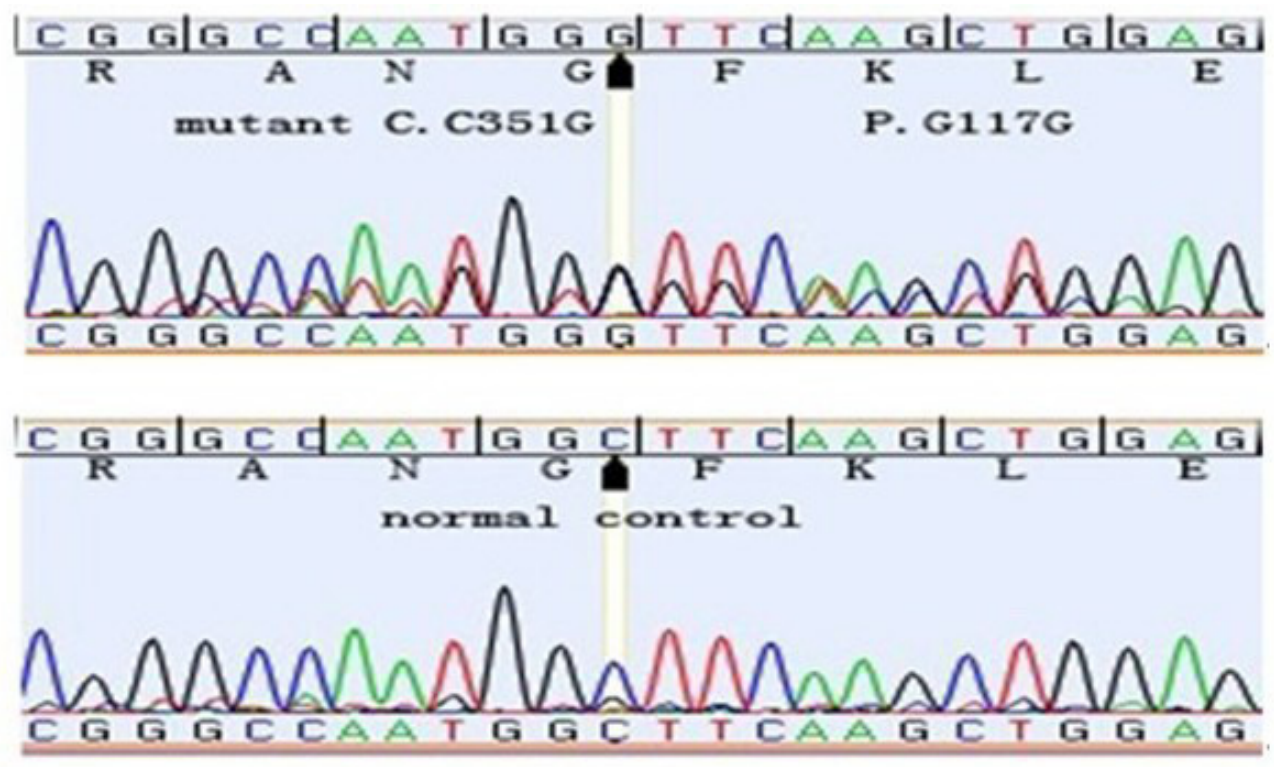

Figure 4. Patient 3 c.351CG(p.G117G). 
C CACACCGCTGGCTCTGCATGGTTCATT T T C mutant exon7 $1655 \mathrm{C} \rightarrow \mathrm{T}$

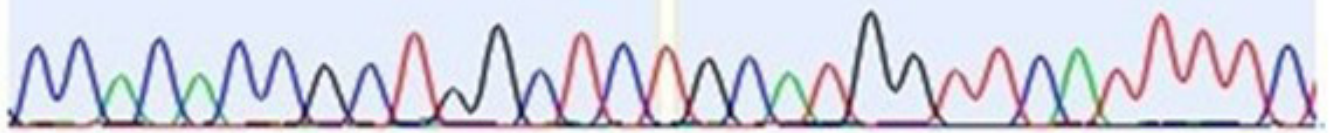

C CACACCGCTGGCTCCGCATGGTTCATTTTC normal control

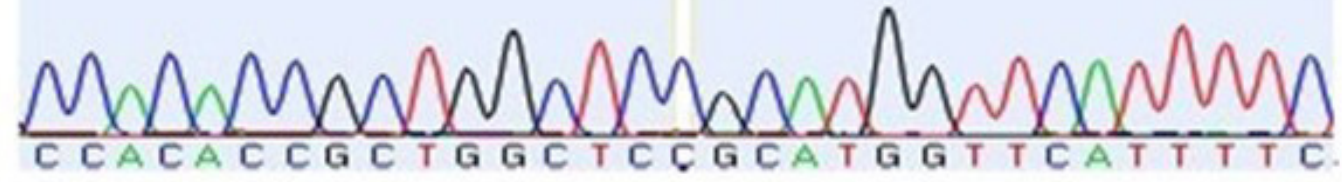

Figure 5. Patient 6 NR5A1 exon 7 noncoding region $1655 \mathrm{C} \rightarrow \mathrm{T}$.

TCTCAGTACCTCCCCCCCCCGCCCCCTGAAACA mutant exon7 $2973 \quad \mathrm{~T} \rightarrow \mathrm{C}$

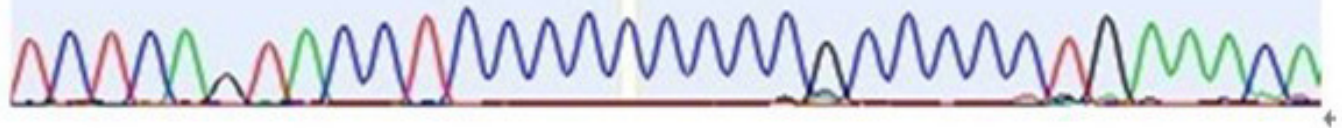

TCT CAGTACCTCC C C TC C C C GC C C C T GAAACA normal control

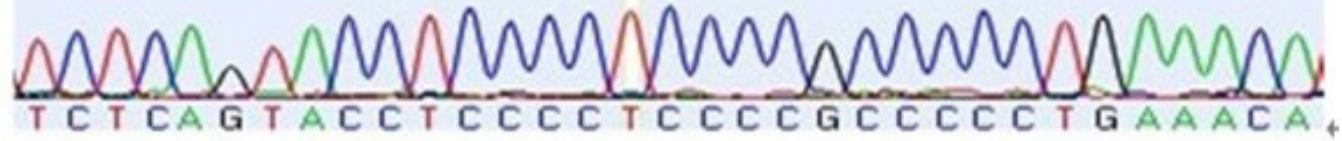

Figure 6. Patient 7 NR5A1 exon 7 noncoding region $2973 \mathrm{~T} \rightarrow \mathrm{C}$. 


\section{Cohort analysis}

PIN1 and NR5A1 mutations were identified in 2 and 5 of the 50 patients studied, respectively. An overview of these changes and associated clinical phenotypes is provided in Table 2. Notably, these changes were found only in patients with inappropriately low serum concentrations of LH and FSH and testosterone or estradiol. NR5A1 mutations were found in the cohort of patients with primary amenorrhea and uterine or ovarian hypoplasia.

Table 2. Genetic, clinical, and biochemical features of patients with SF1 and PIN1 mutations reported here.

\begin{tabular}{|c|c|c|c|c|c|c|c|}
\hline & \multicolumn{7}{|c|}{ Patient } \\
\hline & 1 & 2 & 3 & 4 & 5 & 6 & 7 \\
\hline Amino acid change & Ser32Cys & Leu245Trp & Gly117Gly & Gly146Ala & Gly146Ala & & \\
\hline Exon or intron & Pin1 exon2 & sflexon4 & sflexon4 & sflexon4 & sflexon4 & sflexon7 & sflexon7 \\
\hline Nucleotide change & c. $94 \mathrm{~A}>\mathrm{T}$ & c. $734 \mathrm{~T}>\mathrm{G}$ & c. $351 \mathrm{C}>\mathrm{G}$ & c. $437 \mathrm{G}>\mathrm{C}$ & c. $437 \mathrm{G}>\mathrm{C}$ & e. $1655 \mathrm{C}>\mathrm{T}$ & e. $2973 \mathrm{~T}>\mathrm{C}$ \\
\hline Karyotype & XY & XY & XY & XX & XY & XX & XX \\
\hline Phenotype & Male & Male & Male & Female & Male & Female & Female \\
\hline Age (years) & 19 & 20 & 22 & 20 & 18 & 22 & 17 \\
\hline Additional feature & & & & & & No uterus and ovary & \\
\hline \multicolumn{8}{|l|}{$\mathrm{LH}(\mathrm{mu} / \mathrm{mL})$} \\
\hline Basal & 0.135 & 0.45 & 0.24 & 0.29 & 0.112 & 0.278 & $<0.10$ \\
\hline Peak & 1.45 & 5.21 & 6.86 & 0.68 & 2.22 & 0.697 & 2.18 \\
\hline \multicolumn{8}{|l|}{$\mathrm{FSH}(\mathrm{mu} / \mathrm{mL})$} \\
\hline Basal & 0.32 & 1.16 & 1.12 & 0.59 & 0.466 & 0.944 & 0.18 \\
\hline Peak & 3.22 & 4.14 & 6.68 & 1.18 & 3.25 & 1.84 & 2.91 \\
\hline Testosterone (nM) & 2.73 & $<0.69$ & 1.15 & $<0.69$ & $<0.69$ & 1.24 & $<0.69$ \\
\hline Estradiol (pg/mL) & $<20.0$ & $<20.0$ & $<20.0$ & 20.0 & $<20.0$ & 25.1 & $<20.0$ \\
\hline $\mathrm{ACTH}(\mathrm{pg} / \mathrm{mL})$ & 35 & 11.9 & 23.9 & 45.6 & 21.1 & 38.1 & 0.99 \\
\hline Cortisol (nM) & 372 & 195 & 317 & 91 & 317 & 328 & $<10.0$ \\
\hline An $(n M)$ & 7.49 & 3.08 & 4.8 & $<0.10$ & 1.68 & 3.75 & 237 \\
\hline DHEAS (nM) & 6270 & 4070 & 3040 & 470 & 2560 & 1380 & 2050 \\
\hline 17-OH-P (nM) & 3.48 & 0.97 & 1.06 & 0.39 & 0.94 & 0.91 & 4.45 \\
\hline
\end{tabular}

\section{Case histories}

All 7 patients were underwent hormone measurement, instrumental evaluation, medical history, and physical exam. Patients $1,2,3$, and 5 presented a small phallus $(<3 \mathrm{~cm})$, small pre-pubertal testicular volume $(<4 \mathrm{~mL})$, abnormal sexual maturation, and incomplete development of secondary sexual characteristics (e.g., facial and axillary hair growth and deepening of the voice). KS is diagnosed in patient 3 whose low serum gonadotropins and gonadal steroids were coupled with a compromised sense of smell. Patients 4,6 , and 7 presented as a young adult with primary amenorrhea, who had normal female external genital. Patients 4 and 7 showed normal development of breasts, but patient 6 showed no breast development and uterus and ovary were not identified on ultrasound. The karyotype was 46,XY in male patients and 46,XX in female patients. Endocrine evaluation at diagnosis showed low-basal testosterone levels in all male patients and subnormal LH and FSH concentrations in all patients. Of note, 17-hydroxyprogesterone, DHEAS, and basal cortisol were within normal ranges in each patient and no symptoms or signs of adrenal insufficiency have emerged in any of these patients during follow up. Further investigation of the hypothalamic-pituitary-gonadal (HPG) endocrine axis in these patients suggested hypogonadotropic hypogonadism in additional to a primary gonadotropic defect. Testosterone supplementation was given, which resulted in a 
good response in penile growth. The goal of estrogen treatment includes the development of breast tissue, attainment of appropriate stature, induction of menses, growth of the uterus for possible reproductive function, and maintenance of skeletal health.

\section{DISCUSSION}

Although puberty is thought to be controlled by a network of transcriptional modules, the mechanisms controlling puberty remain largely unknown. Once normal puberty is initiated, it is generally completed in 3 to 4 years through a progression of events (Layman, 2007). IHH is a rare disease that is defined by reduced synthesis and secretion of steroid hormones due to low LH and FSH secretion, resulting in the failed induction of puberty. The HPG axis differs from other endocrine axes involving the anterior pituitary gland, because gonadotropin-releasing hormone $(\mathrm{GnRH})$-producing neurons originate outside the central nervous system in the olfactory placode and migrate to the mediobasal hypothalamus. Further, the HPG axis is complex, featuring both negative and positive feedback by sex steroids (Ojeda et al., 2010). Therefore, this sophisticated system is inherently susceptible to genetic defects, resulting in pubertal and/or reproductive failure. Mutations in a number of genes have been identified in patients with primary genetic IHH. However, currently known genetic defects account for only $30 \%$ of all IHH cases. Despite recent advances in the field, the genetic and molecular mechanisms of mutation genes remain unknown, and there is room for further discovery. These genes encode proteins that regulate GnRH neuronal development, migration from the nasal placode to the hypothalamus, GnRH secretion, or GnRH action (Wray et al., 1989; Schwanzel-Fukuda, 1999; Balasubramanian et al., 2010). Some genetic mutations are sufficient to cause IHH; in other cases, IHH results from the combination of more than one genetic abnormality.

$S F 1$ regulates the transcription of several enzymes involved in steroid/androgen biosynthesis. $S F 1$ is also a transcription factor that plays a pivotal role in adrenal and reproductive function, and it influences gene transcription at multiple levels and at different stages of development. In mice, complete loss of SF1 function results in apoptosis of the developing adrenal gland and gonad during early embryogenesis (Luo et al., 1994; Sadovsky et al., 1995). SF1 plays an important role in multiple aspects of testicular and ovarian development, integrity and function, as it regulates a number of critical genes involved in these processes. Indeed, a milder loss-of-function change in $S F 1$ has been associated with micropenis, undescended testes, and anomalies of ovarian development and function (Wada et al., 2005, 2006; Philibert et al., 2007). The initial search for changes in $S F 1$ among humans focused on patients with combined gonadal and adrenal failure (Achermann et al., 2002); however, more recent studies have shown that heterozygous $S F 1$ mutations are relatively common in 46,XY patients with mild or partial gonadal dysgenesis, impaired androgenization, and apparently normal adrenal function (Lin et al., 2007; Kohler et al., 2008). These mutations are also common in 46,XX girls or women with ovarian insufficiency (Coutant et al., 2007; Lourenco et al., 2009).

PIN1 plays a role in transcription of gonadotropin-subunit genes. Through a phosphorylation-regulated pathway, it also promotes $S F 1$ ubiquitination, which facilitates the interaction between $S F 1$ and Pitx 1 and results in an enhancement of $S F 1$ transcriptional activity (Zhao et al., 2001). In gonadotropic cells, sufficient levels of activated PIN1 are maintained through transcriptional and post-translational regulation by GnRH-induced signaling cas- 
cades. PIN1 activity is regulated by $G n R H$, indicating that it is a novel player in the GnRHsignaling pathway and gonadotropin gene expression (Lu et al., 2002). PIN1 is involved in transcription of the gonadotropin $\beta$-subunit by modulating the activity of various transcription factors. PIN1 has been found at the promoters of both gonadotropin $\beta$-subunit genes and is presumably recruited by its interaction with SF1, Pitx1, and/or Egr-1 (Chen et al., 2007; Benayoun and Veitia, 2009). In some cases, there is functional synergy, although this appears to be promoter-context specific. The functional interaction with $S F 1$ on the $L H \beta$ gene was most striking (Walsh and Shupnik, 2009).

In conclusion, we identified a series of missense and synonymous mutations in the NR5A1 and PIN1 genes that are associated with primary amenorrhea and male IHH with apparently normal adrenal function. Whether these patients will develop adrenal insufficiency over time remains to be investigated. Thus, determining the genetic cause in patients with IHH may be useful for future research, counseling families of affected patients, long-term followup, developing a diagnostic genetic test, and future treatment of IHH.

\section{ACKNOWLEDGMENTS}

Research supported by the 12th Five-year Plan Key Science Project (\#2012BAI09B04), the Ministry of Health Industry Fund (\#201002006) and PCSIRT1131.

\section{REFERENCES}

Achermann JC, Meeks JJ and Jameson JL (2001). Phenotypic spectrum of mutations in DAX-1 and SF-1. Mol. Cell. Endocrinol. 185: 17-25.

Achermann JC, Ozisik G, Ito M, Orun UA, et al. (2002). Gonadal determination and adrenal development are regulated by the orphan nuclear receptor steroidogenic factor-1, in a dose-dependent manner. J. Clin. Endocrinol. Metab. 87: 1829-1833.

Atchison FW and Means AR (2003). Spermatogonial depletion in adult Pin1-deficient mice. Biol. Reprod. 69: 1989-1997.

Balasubramanian R, Dwyer A, Seminara SB, Pitteloud N, et al. (2010). Human GnRH deficiency: a unique disease model to unravel the ontogeny of GnRH neurons. Neuroendocrinology 92: 81-99.

Benayoun BA and Veitia RA (2009). A post-translational modification code for transcription factors: sorting through a sea of signals. Trends Cell Biol. 19: 189-197.

Chen WY, Weng JH, Huang CC and Chung BC (2007). Histone deacetylase inhibitors reduce steroidogenesis through SCF-mediated ubiquitination and degradation of steroidogenic factor 1 (NR5A1). Mol. Cell. Biol. 27: 7284-7290.

Coutant R, Mallet D, Lahlou N, Bouhours-Nouet N, et al. (2007). Heterozygous mutation of steroidogenic factor-1 in 46,XY subjects may mimic partial androgen insensitivity syndrome. J. Clin. Endocrinol. Metab. 92: 2868-2873.

Crowley WF Jr, Pitteloud N and Seminara S (2008). New genes controlling human reproduction and how you find them. Trans. Am. Clin. Climatol. Assoc. 119: 29-37.

Hardelin JP and Dode C (2008). The complex genetics of Kallmann syndrome: KAL1, FGFR1, FGF8, PROKR2, PROK2, et al. Sex. Dev. 2: 181-193.

Ingraham HA, Lala DS, Ikeda Y, Luo X, et al. (1994). The nuclear receptor steroidogenic factor 1 acts at multiple levels of the reproductive axis. Genes Dev. 8: 2302-2312.

Kohler B, Lin L, Ferraz-de-Souza B, Wieacker P, et al. (2008). Five novel mutations in steroidogenic factor 1 (SF1, NR5A1) in 46,XY patients with severe underandrogenization but without adrenal insufficiency. Hum. Mutat. 29: 59-64.

Layman LC (2007). Hypogonadotropic hypogonadism. Endocrinol. Metab. Clin. North Am. 36: 283-296.

Lin L, Philibert P, Ferraz-de-Souza B, Kelberman D, et al. (2007). Heterozygous missense mutations in steroidogenic factor 1 (SF1/Ad4BP, NR5A1) are associated with 46,XY disorders of sex development with normal adrenal function. J. Clin. Endocrinol. Metab. 92: 991-999.

Lourenco D, Brauner R, Lin L, De Perdigo A, et al. (2009). Mutations in NR5A1 associated with ovarian insufficiency. 
N. Engl. J. Med. 360: 1200-1210.

Lu PJ, Zhou XZ, Liou YC, Noel JP, et al. (2002). Critical role of WW domain phosphorylation in regulating phosphoserine binding activity and Pin1 function. J. Biol. Chem. 277: 2381-2384.

Luo X, Ikeda Y and Parker KL (1994). A cell-specific nuclear receptor is essential for adrenal and gonadal development and sexual differentiation. Cell 77: 481-490.

Ojeda SR, Dubay C, Lomniczi A, Kaidar G, et al. (2010). Gene networks and the neuroendocrine regulation of puberty. Mol. Cell. Endocrinol. 324: 3-11.

Philibert P, Zenaty D, Lin L, Soskin S, et al. (2007). Mutational analysis of steroidogenic factor 1 (NR5a1) in 24 boys with bilateral anorchia: a French collaborative study. Hum. Reprod. 22: 3255-3261.

Sadovsky Y, Crawford PA, Woodson KG, Polish JA, et al. (1995). Mice deficient in the orphan receptor steroidogenic factor 1 lack adrenal glands and gonads but express P450 side-chain-cleavage enzyme in the placenta and have normal embryonic serum levels of corticosteroids. Proc. Natl. Acad. Sci. U. S. A. 92: 10939-10943.

Schimmer BP and White PC (2010). Minireview: steroidogenic factor 1: its roles in differentiation, development, and disease. Mol. Endocrinol. 24: 1322-1337.

Schwanzel-Fukuda M (1999). Origin and migration of luteinizing hormone-releasing hormone neurons in mammals. Microsc. Res. Tech. 44: 2-10.

Shinoda K, Lei H, Yoshii H, Nomura M, et al. (1995). Developmental defects of the ventromedial hypothalamic nucleus and pituitary gonadotroph in the Ftz-F1 disrupted mice. Dev. Dyn. 204: 22-29.

Wada Y, Okada M, Hasegawa T and Ogata T (2005). Association of severe micropenis with Gly146Ala polymorphism in the gene for steroidogenic factor-1. Endocr. J. 52: 445-448.

Wada Y, Okada M, Fukami M, Sasagawa I, et al. (2006). Association of cryptorchidism with Gly146Ala polymorphism in the gene for steroidogenic factor-1. Fertil. Steril. 85: 787-790.

Walsh HE and Shupnik MA (2009). Proteasome regulation of dynamic transcription factor occupancy on the GnRHstimulated luteinizing hormone beta-subunit promoter. Mol. Endocrinol. 23: 237-250.

Wray S, Nieburgs A and Elkabes S (1989). Spatiotemporal cell expression of luteinizing hormone-releasing hormone in the prenatal mouse: evidence for an embryonic origin in the olfactory placode. Brain Res. Dev. Brain Res. 46: 309318.

Zhao L, Bakke M and Parker KL (2001). Pituitary-specific knockout of steroidogenic factor 1. Mol. Cell. Endocrinol. 185: 27-32. 